\title{
Impact of stirring speed, glycerin and sodium chloride concentrations on photoprotective nanoemulsions
}

\author{
Impacto da velocidade de agitação, contração de glicerina e de cloreto de sódio em \\ nanoemulsões fotoprotetoras
}

\author{
Débora Granemann e Silva1, Camila Areias de Oliveira ${ }^{1}$, Daniela D'Almeida Peres ${ }^{1}$, Margarida Pereira ${ }^{2}$, \\ Nicole Pereira ${ }^{2}$, Tânia Santos Almeida ${ }^{2,3}$, Joana Portugal Mota ${ }^{2}$, Catarina Rosado ${ }^{2}$, Vânia Rodrigues Leite- \\ Silva $^{4}$, Maria Valéria Robles Velasco ${ }^{1}$, André Rolim Baby ${ }^{1}$ \\ 'Faculdade de Ciências Farmacêuticas, Universidade de São Paulo. São Paulo, Brasil \\ ${ }^{2} \mathrm{CBios}$ - Research Center for Biosciences and Health Technologies, Universidade Lusófona, Lisboa, Portugal \\ ${ }^{3}$ Centro de Química e Bioquímica / Departamento de Química e Bioquímica, Faculdade de Ciências, Universidade de \\ Lisboa, Lisboa, Portugal \\ ${ }^{4}$ Instituto de Ciências Ambientais Químicas e Farmacêuticas, Universidade Federal de São Paulo, Diadema SP, Brasil. \\ Diadema, SP, Brasil \\ Email: andrerb@usp.br \\ Fontes de Financiamento: FAPESP e CNPq
}

\begin{abstract}
New technologies that improve the physical as the sensory properties of sunscreens can help to increase its continued use and impact on health. The use of nanoemulsions in the development of photoprotective vehicles is an advantage, since nanostructured components may have superior properties regarding their performance when compared to conventional products. The advantages of using nanobiotechnology in manufacture of cosmetic and dermatological formulations arise from the protection of compounds from chemical or enzymatic degradation, from the control of their release, and also to the prolonged retention time of cosmetic ingredients in the stratum corneum. Thus, this study aimed to evaluate the impact of stirring speed and of glycerin and sodium chloride concentrations in the development and effectiveness of a nanoemulsion containing ethylhexyl methoxycinnamate and benzophenone-3. The results of statistical analyses regarding the impact of the variables in the process of nanoemulsion development showed that these parameters affect the phase inversion temperature (PIT). However, this did not affect the particle size and the photoprotective efficacy in vitro.
\end{abstract}

Key-words: Nanoemulsion; Photoprotection; Ethylhexyl methoxycinnamate; Benzophenone-3

\section{Resumo}

Novas tecnologias que melhorem a aparência física e o sensorial de fotoprotetores podem ajudar a aumentar a continuidade do uso e o seu impacto em saúde. A utilização de nanoemulsões como veículos cosméticos para o desenvolvimento de fotoprotetores, torna-se uma vantagem, pois componentes nanoestruturados podem apresentar propriedades superiores quanto a sua performance em comparação a produtos convencionais. Do ponto de vista científico, as vantagens do uso da nanobiotecnologia na produção de nanocosméticos e formulações dermatológicas são concernentes à proteção de compostos quanto à degradação química ou enzimática, ao controle de sua liberação e ao prolongamento do tempo de retenção dos ativos cosméticos no estrato córneo. Este estudo pretende avaliar a influência de diversas variáveis no processo de desenvolvimento e avaliação da eficácia fotoprotetora in vitro de uma nanoemulsão contendo p-metoxicinamato de octila e benzofenona-3, obtida empregando o método da temperatura de inversão de fases (PIT). Os resultados demonstram que a velocidade de agitação, a concentração de glicerina e a do cloreto de sódio influenciam a temperatura em que a emulsão inverte de A/O para O/A (PIT) das formulações compostas de p-metoxicinamato de octila e benzofenona-3, entretanto, não influenciam o tamanho de partícula nem a eficácia fotoprotetora in vitro.

Palavras-chave: Nanoemulsão; Fotoproteção; p-metoxicinamato de octila; Benzofenona-3. 


\section{Introduction}

Moderate sun exposure is beneficial to humans, promoting the physical and mental welfare and stimulating the melanin production. However, solar radiation can also cause damage to the human body ${ }^{[1]}$. The incidence of skin cancer has steadily increased and the main cause is excessive exposure to solar radiation. Thus, the widespread use of sunscreens has been indicated by several international institutions as the most appropriate method to reduce the damage caused by solar radiation, particularly skin cancer and premature aging ${ }^{[2]}$.

Fluid and semi-solid emulsions have been widely used in cosmetics as vehicles for active compounds [3]. Nanoemulsions are preparations with particle size in the nanometer scale, metastable, often fluid, with a bluish gloss and appearance that can vary from translucent to opaque, depending on the particle size ${ }^{[4,5]}$.

The vast use of nanoemulsions in both cosmetics and pharmaceutical industries, along with the growing concern over the damages caused by excessive exposure to solar radiation, justifies the selection of these vehicles for pharmacotechnical studies. In agreement with this scenario, this study involved the development of photoprotective nanoemulsions employing a low energy process. Furthermore, the particle size and the in vitro effectiveness of the photoprotective systems was evaluated, considering the possible influences of stirring speed, glycerin and sodium chloride concentrations in the development process ${ }^{[6,7]}$.

\section{Material and Methods}

\section{Formulations}

A factorial experimental design (DoE, 33, Minitab ${ }^{\circledR}$ ) was defined, with the selection of three variables at three different levels, as shown in Table 1. The qualitative and quantitative composition (in $\mathrm{cg} / \mathrm{g}$ ) of the nanoemulsions is shown in Table 2.

\section{Formulations Characterization}

Phase Inversion Temperature (PIT) determination

The temperature of the hydrophilic-lipophilic balance (HLB), also defined as the PIT, was determined by the electrical conductivity method. Thus, all components (Table 2) were weighed in a glass beaker and heated gradually with mechanic stirring (at $400 \mathrm{rpm}$ ), while the conductivity (in $\mathrm{mS}$ ) was measured as a function of temperature. The conductivity measurements were

\section{Introdução}

A exposição moderada ao sol traz benefícios ao ser humano, como sensação de bem estar físico, mental e estímulo à produção de melanina. Porém, o excesso de radiação solar também pode causar prejuízos ao organismo ${ }^{[1]}$. A incidência de câncer de pele têm aumentado continuamente e a principal causa é o excesso de exposição a radiação solar. Desta forma, o uso em larga escala de protetores solares tem sido indicado por diversas instituições internacionais como método apropriado para reduzir os danos causados pela radiação solar, especialmente o câncer de pele e o envelhecimento precoce ${ }^{[2]}$.

Emulsões fluídas e semi-sólidas vêm sendo amplamente usadas em cosméticos como veículos para compostos ativos ${ }^{[3]}$. Nanoemulsões são preparações com tamanho de partícula em escala nanométrica, metaestáveis, geralmente fluídas, com brilho azulado e aspecto que pode variar de translúcido a opaco, dependendo do tamanho das partículas da fase interna ${ }^{[4,5]}$.

O amplo emprego das nanoemulsões em indústrias cosméticas e farmacêuticas assim como a crescente preocupação com os danos causados pelo excesso de exposição à radiação solar justificam sua escolha como tema de estudos farmacotécnicos. Em concordância com este panorama, o presente trabalho envolveu o desenvolvimento de nanoemulsões fotoprotetoras empregando um método de baixa energia. Adicionalmente, este trabalho avaliou o tamanho de partícula e a eficácia fotoprotetora in vitro dos sistemas obtidos, considerando a possível influência de três variáveis (velocidade de agitação, concentração de glicerina e concentração de cloreto de sódio) no processo de desenvolvimento da formulação ${ }^{[6,7]}$

\section{Materiais e Métodos}

\section{Formulações}

Com o objetivo de otimizar o processo de desenvolvimento da nanoemulsão, foi elaborado um planejamento experimental do tipo fatorial $\left(D O E, 3^{3}\right.$, Minitab $\left.{ }^{\circledR}\right)$ selecionando três variáveis em três diferentes níveis, conforme Tabela 1. A Tabela 2 apresenta a composição qualitativa e quantitativa (em $\mathrm{cg} / \mathrm{g}$ ) das nanoemulsões analisadas.

\section{Caracterização das Formulações}

Determinação da Temperatura de Inversão de Fases (PIT)

A temperatura de equilíbrio hidrofílico-lipofílico (EHL), também definida com temperatura de inversão de fases (PIT) foi determinada pelo método da condutividade elétrica. Dessa forma, todos os componentes 
recorded at room temperature, i.e. $22.0 \pm 2.0){ }^{\circ} \mathrm{C}$, and at $5.0{ }^{\circ} \mathrm{C}$ increments up to $80.0^{\circ} \mathrm{C}$. The PIT was determined when a marked decrease in the conductivity value was observed, which evidenced the emulsion inversion from oil in water $(\mathrm{O} / \mathrm{W})$ to water in oil $(\mathrm{W} / \mathrm{O})$ $[8 ; 9 ; 10]$.

\section{$\underline{\text { Particle size determination }}$}

The particle size and size distribution were measured with an acoustic spectrometer (DT-1200, Dispersion Technology Inc.). The nanoemulsions were prepared, stored for 24 hours and transferred, without dilution, to a specific compartment of the instrument. Analyses were performed in triplicate, in the distance range of $0.325 \mathrm{~mm}$ to $20.000 \mathrm{~mm}$ between the transmitter and the receiver, and in the ${ }^{[3 ; 100]} \mathrm{MHz}$ frequency range. The sound attenuation was monitored over time ${ }^{[11 ; 12 ; 13 ; 14]}$.
(Tabela 2) foram pesados em béquer de vidro e gradualmente aquecidos sob agitação mecânica (400 rpm) enquanto a condutividade (em $\mathrm{mS}$ ) foi medida em função da temperatura. As leituras de condutividade foram registradas a partir da temperatura ambiente i.e. $22,0 \pm$ $2,0^{\circ} \mathrm{C}$ e a cada incremento de $5,0^{\circ} \mathrm{C}$ até atingir 80,0 ${ }^{\circ} \mathrm{C}$. A PIT foi determinada quando ocorreu diminuição acentuada no valor da condutividade, o que evidenciou a inversão da emulsão de óleo em água $(\mathrm{O} / \mathrm{A})$ para água em óleo $(\mathrm{A} / \mathrm{O})^{[8 ; 9 ; 10]}$.

Determinação do Tamanho de Partícula

O tamanho de partícula e a distribuição de tamanhos foram medidas por espectrômetro acústico (DT-1200, Dispersion Technology Inc., Bedford Hills, NY). Para determinação do tamanho de partícula e da distribuição de tamanhos, uma amostra de nanoemulsão foi preparada, armazenada durante 24 horas e então transferida para o compartimento específico do equipamento DT-1200 sem diluição. As análises foram realizadas em triplicata, no intervalo de distâncias ${ }^{[0,325 ; 20]} \mathrm{mm}$ entre transmissor e receptor e na faixa de frequências [3; 100] MHz. A atenuação do som foi monitorada durante o tempo ${ }^{[11 ; 12 ; 13 ; 14]}$.

Table 1/ Tabela 1. Levels and Factors in the design of experiments $3^{3}$ (DOE, Minitab $^{\circledR}$ ) for the development of nanoemulsions

Planejamento fatorial $3^{3}\left(D O E\right.$, Minitab $\left.{ }^{\circledR}\right)$ para otimização do processo de desenvolvimento da nanoemulsão

\begin{tabular}{cccc}
\hline Factors/ Variável & Level/ Nível (-) & Level/ Nível (0) & Level/ Nível (+) \\
\hline Speed/ Velocidade de & 400 & 600 & 800 \\
agitação (rpm) & & & 3 \\
Sodium chloride/ NaCl (cg/g) & 1 & 2 & 15 \\
Glycerin/ Glicerina (cg/g) & 5 & 10 & \\
\hline
\end{tabular}


Table 2. Qualitative and quantitative $(\mathrm{cg} / \mathrm{g})$ composition of the formulations

\begin{tabular}{|c|c|c|c|c|}
\hline \multirow{3}{*}{$\begin{array}{l}\text { Formulation } \\
\text { Codes }\end{array}$} & \multicolumn{4}{|c|}{ Components } \\
\hline & \multirow{2}{*}{$\begin{array}{l}\text { Fixed Components } \\
\qquad(\mathrm{cg} / \mathrm{g})\end{array}$} & \multicolumn{3}{|c|}{ Variable Components } \\
\hline & & $\begin{array}{r}\text { Speed } \\
(\text { rpm) }\end{array}$ & $\begin{array}{l}\text { Glycerin } \\
\text { (cg/g) }\end{array}$ & $\begin{array}{l}\text { Sodium chloride } \\
\text { (cg/g) }\end{array}$ \\
\hline 01 & & 800 & 15 & 2 \\
\hline 02 & & 800 & 10 & 3 \\
\hline 03 & & 400 & 10 & 2 \\
\hline 04 & & 400 & 5 & 2 \\
\hline 05 & \multirow{2}{*}{$\mathrm{BHT}(0.1)$} & 800 & 15 & 1 \\
\hline 06 & & 800 & 5 & 3 \\
\hline 07 & $\mathrm{EDTA} \mathrm{Na}_{2}(0.1)$ & 600 & 5 & 3 \\
\hline 08 & \multirow{5}{*}{$\begin{array}{l}\text { Phenoxyethanol (and) } \\
\text { methylparaben (and) } \\
\text { ethylparaben (and) } \\
\text { propylparaben (and) } \\
\text { butylparaben (and) } \\
\text { isobutylparaben }(0.5)\end{array}$} & 400 & 15 & 2 \\
\hline 09 & & 400 & 5 & 1 \\
\hline 10 & & 600 & 10 & 1 \\
\hline 11 & & 400 & 15 & 3 \\
\hline 12 & & 600 & 5 & 2 \\
\hline 13 & Cetearyl alcohol (3.0) & 800 & 10 & 2 \\
\hline 14 & \multirow{2}{*}{ Acqua (q.s.p.) } & 400 & 10 & 3 \\
\hline 15 & & 600 & 15 & 3 \\
\hline 16 & \multirow{2}{*}{$\begin{array}{l}\text { Ethylhexyl methoxycinnamate } \\
\qquad(7.5)\end{array}$} & 800 & 10 & 1 \\
\hline 17 & & 600 & 15 & 1 \\
\hline 18 & Benzophenone-3 (2.0) & 600 & 5 & 1 \\
\hline 19 & \multirow{2}{*}{$\begin{array}{l}\text { Caprylic/capric triglyceride } \\
\qquad(5.0)\end{array}$} & 400 & 5 & 3 \\
\hline 20 & & 400 & 15 & 1 \\
\hline 21 & Oleth-3 (2.0) & 800 & 5 & 2 \\
\hline 22 & \multirow{6}{*}{ Oleth-20 (10.0) } & 600 & 15 & 2 \\
\hline 23 & & 800 & 15 & 3 \\
\hline 24 & & 800 & 5 & 1 \\
\hline 25 & & 400 & 10 & 1 \\
\hline 26 & & 600 & 10 & 2 \\
\hline 27 & & 600 & 10 & 3 \\
\hline
\end{tabular}


Tabela 2. Composição quali e quantitativa $(\mathrm{cg} / \mathrm{g})$ das amostras elaboradas no planejamento experimental do tipo fatorial, $3^{3}\left(D O E\right.$, Minitab $\left.{ }^{\circledR}\right)$, para otimização do processo de desenvolvimento da nanoemulsão

\begin{tabular}{|c|c|c|c|c|}
\hline \multirow{3}{*}{ Formulações } & \multicolumn{4}{|c|}{ Composição } \\
\hline & \multirow{2}{*}{$\begin{array}{c}\text { Componentes Fixos } \\
(\mathrm{cg} / \mathrm{g})\end{array}$} & \multicolumn{3}{|c|}{ Componentes Variáveis } \\
\hline & & $\begin{array}{l}\text { Agitação } \\
\text { (rpm) }\end{array}$ & $\begin{array}{c}\text { Glicerina } \\
(\mathbf{c g} / \mathrm{g})\end{array}$ & $\begin{array}{c}\text { Cloreto de sódio } \\
\text { (cg/g) }\end{array}$ \\
\hline 01 & & 800 & 15 & 2 \\
\hline 02 & & 800 & 10 & 3 \\
\hline 03 & & 400 & 10 & 2 \\
\hline 04 & & 400 & 5 & 2 \\
\hline 05 & & 800 & 15 & 1 \\
\hline 06 & BHT $(0,1)$ & 800 & 5 & 3 \\
\hline 07 & & 600 & 5 & 3 \\
\hline 08 & EDTA Na $2(0,1)$ & 400 & 15 & 2 \\
\hline 09 & Phenoxyethanol (and) & 400 & 5 & 1 \\
\hline 10 & $\begin{array}{l}\text { methylparaben (and) } \\
\text { ethylparaben (and) propylparaben }\end{array}$ & 600 & 10 & 1 \\
\hline 11 & (and) butylparaben (and) & 400 & 15 & 3 \\
\hline 12 & isobutylparaben $(0,5)$ & 600 & 5 & 2 \\
\hline 13 & Cetearyl alcohol $(3,0)$ & 800 & 10 & 2 \\
\hline 14 & Acqua (as.p.) & 400 & 10 & 3 \\
\hline 15 & & 600 & 15 & 3 \\
\hline 16 & Ethylhexyl methoxycinnamate & 800 & 10 & 1 \\
\hline 17 & & 600 & 15 & 1 \\
\hline 18 & Benzophenone-3 $(2,0)$ & 600 & 5 & 1 \\
\hline 19 & Caprylic/capric triglyceride $(5,0)$ & 400 & 5 & 3 \\
\hline 20 & Oleth $-3(20)$ & 400 & 15 & 1 \\
\hline 21 & & 800 & 5 & 2 \\
\hline 22 & Oleth-20 $(10,0)$ & 600 & 15 & 2 \\
\hline 23 & & 800 & 15 & 3 \\
\hline 24 & & 800 & 5 & 1 \\
\hline 25 & & 400 & 10 & 1 \\
\hline 26 & & 600 & 10 & 2 \\
\hline 27 & & 600 & 10 & 3 \\
\hline
\end{tabular}

Composição qualitativa descrita segundo nomenclatura INCI (International Nomenclature of Cosmetic Ingredients 
Determination of Effectiveness photoprotection in vitro

The in vitro photoprotective effectiveness was determined by use of a diffuse reflectance spectrophotometer equipped with an integrating sphere (Ultraviolet Transmittance Analyzer, UV-2000S, Labsphere). The nanoemulsions were prepared, stored for 24 hours and uniformly applied to the rough side of a PMMA plate (polymethylacrylate - Helioplate ${ }^{\circledR} \mathrm{HD}$, HelioScreeen), with an application rate of 0.75 milligrams of nanoemulsion per square centimetre. The film formed on the plate was allowed to dry under ambient room temperature conditions at (22.0 \pm 2.0) $\mathrm{C}$ for 30 minutes and the UV absorbance was measured at nine different points of the PMMA plate. The resulting absorbance was converted by the equipment's software into in vitro sun protection factor (SPF) values ${ }^{[15 ; 16 ; 17]}$.

\section{Statistical Analysis}

A factorial experimental design $\left(\operatorname{DoE} 3^{3}\right.$, $\left.\mathrm{Minitab}^{\circledR}\right)$ was employed to analyze the influence of stirring speed, glycerin and sodium chloride concentrations in the nanoemulsions' development process. The results obtained were analyzed by a specific software $\left(\right.$ Minitab $^{\circledR}$, version 15$)$, with a significance level of $0.05(p<0.05)$, for the determination of statistically significant results ${ }^{[18 ; 19]}$.

Table 3. PIT, particle size and in vitro sun protection factor obtained by the design of experiments $3^{3}$ for nanoemulsions

\begin{tabular}{|c|c|c|c|c|c|c|c|c|}
\hline $\begin{array}{c}\text { Formulation } \\
\text { Codes }\end{array}$ & $\begin{array}{l}\text { Speed } \\
\text { (rpm) }\end{array}$ & $\begin{array}{c}\text { Glycerin } \\
\text { (cg/g) }\end{array}$ & $\begin{array}{c}\text { Sodium } \\
\text { chloride } \\
\text { (cg/g) }\end{array}$ & $\begin{array}{l}P I T \\
\left({ }^{\circ} \mathrm{C}\right)\end{array}$ & $\begin{array}{l}\text { Size1 } \\
(\mu \mathrm{m})\end{array}$ & $\begin{array}{c}\text { Size2 } \\
(\mu \mathrm{m})\end{array}$ & vrf2/vrf & SPF \\
\hline 1 & 800 & 15 & 2 & 59,0 & & & & \\
\hline 2 & 800 & 10 & 3 & 61,0 & 0,639 & 1,331 & 0,24 & 8 \\
\hline 5 & 800 & 15 & 1 & 66,0 & 0,011 & 5,389 & 0,66 & 8 \\
\hline 6 & 800 & 5 & 3 & 64,0 & 0,026 & 4,529 & 0,62 & 8 \\
\hline 7 & 600 & 5 & 3 & 64,0 & 0,010 & 6,283 & 0,75 & 7 \\
\hline 8 & 400 & 15 & 2 & 62,0 & 0,010 & 4,876 & 0,60 & 8 \\
\hline 9 & 400 & 5 & 1 & 72,0 & 0,050 & 9,422 & 0,63 & 8 \\
\hline 14 & 400 & 10 & 3 & 59,0 & 0,011 & 2,163 & 0,30 & 13 \\
\hline 15 & 600 & 15 & 3 & 57,0 & 0,011 & 3,519 & 0,39 & 9 \\
\hline 16 & 800 & 10 & 1 & 66,0 & 0,010 & 0,586 & 0,10 & 19 \\
\hline 17 & 600 & 15 & 1 & 67,0 & 0,011 & 1,572 & 0,19 & 12 \\
\hline 18 & 600 & 5 & 1 & 69,0 & 0,774 & 5,878 & 0,41 & 11 \\
\hline 19 & 400 & 5 & 3 & 66,0 & 0,768 & 2,079 & 0,45 & 14 \\
\hline 20 & 400 & 15 & 1 & 67,0 & 0,010 & 3,178 & 0,70 & 14 \\
\hline 21 & 800 & 5 & 2 & 68,0 & 0,109 & 9,315 & 0,91 & 12 \\
\hline 22 & 600 & 15 & 2 & 62,0 & 0,092 & 9,153 & 0,91 & 10 \\
\hline 23 & 800 & 15 & 3 & 58,0 & 0,120 & 8,951 & 0,91 & 12 \\
\hline
\end{tabular}


Determinação da Eficácia Fotoprotetora in vitro

A eficácia fotoprotetora in vitro foi determinada por espectrofotometria de refletância difusa equipado com esfera de integração (Ultraviolet Transmittance Analyzer, UV-2000S, Labsphere, US). Uma amostra da emulsão foi preparada, armazenada por 24 horas e então alíquotas de $0,75 \mathrm{mg} / \mathrm{cm}^{2}$ de emulsão foram aplicadas uniformemente no lado rugoso da placa de PMMA (Polimetilacrilato - HD Helioplate ${ }^{\circledR}$, HelioScreeen, US) . O filme formado sobre a placa foi deixado para secar sob condições ambientais a $(22,0 \pm 2,0){ }^{\circ} \mathrm{C}$, por 30 minutos. A absorbância UV do filme foi medida em nove pontos diferentes do substrato. A absorbância resultante foi usada para determinar o fator de proteção solar (FPS) in vitro, através do software UV2000S ${ }^{[15 ; 16 ; 17] .}$

\section{Análise Estatística dos Resultados}

No presente estudo, foi realizado planejamento experimental do tipo fatorial, $3^{3}$ (DOE, Minitab ${ }^{\circledR}$ ), para analisar a influência das variáveis no processo de desenvolvimento da nanoemulsão. Os resultados obtidos foram analisados por software específico (Minitab ${ }^{\circledR}$, versão 15$)$, com nível de significância de $5 \%(p<0,05)$ para a determinação de resultados estatisticamente significativos ${ }^{[18 ; 19]}$.

Tabela 3. Valores de PIT, tamanho de partícula e eficácia fotoprotetora in vitro determinados nas amostras do planejamento experimental do tipo fatorial

\begin{tabular}{|c|c|c|c|c|c|c|c|c|}
\hline \multirow[b]{2}{*}{ Formulações } & \multirow[b]{2}{*}{$\begin{array}{l}\text { Agitação } \\
\text { (rpm) }\end{array}$} & \multirow{2}{*}{$\begin{array}{c}\text { Variáveis } \\
\text { Glicerina } \\
(\mathrm{cg} / \mathrm{g})\end{array}$} & \multirow[b]{2}{*}{$\begin{array}{c}\text { Cloreto de } \\
\text { sódio } \\
\text { (cg/g) }\end{array}$} & \multirow[b]{2}{*}{$\begin{array}{l}P I T \\
\left({ }^{\circ} \mathrm{C}\right)\end{array}$} & \multirow[b]{2}{*}{$\begin{array}{c}\text { Tamanho } 1 \\
(\mu \mathrm{m})\end{array}$} & \multirow[b]{2}{*}{$\begin{array}{c}\text { Tamanho } 2 \\
(\mu \mathrm{m})\end{array}$} & \multirow[b]{2}{*}{ vrf2/vrf } & \multirow[b]{2}{*}{ FPS } \\
\hline & & & & & & & & \\
\hline 1 & 800 & 15 & 2 & 59,0 & & & & \\
\hline 2 & 800 & 10 & 3 & 61,0 & 0,639 & 1,331 & 0,24 & 8 \\
\hline 3 & 400 & 10 & 2 & 66,0 & 0,368 & 9,358 & 0,61 & 8 \\
\hline 4 & 400 & 5 & 2 & 67,0 & 0,010 & 2,915 & 0,42 & 7 \\
\hline 5 & 800 & 15 & 1 & 66,0 & 0,011 & 5,389 & 0,66 & 8 \\
\hline 6 & 800 & 5 & 3 & 64,0 & 0,026 & 4,529 & 0,62 & 8 \\
\hline 7 & 600 & 5 & 3 & 64,0 & 0,010 & 6,283 & 0,75 & 7 \\
\hline 8 & 400 & 15 & 2 & 62,0 & 0,010 & 4,876 & 0,60 & 8 \\
\hline 9 & 400 & 5 & 1 & 72,0 & 0,050 & 9,422 & 0,63 & 8 \\
\hline 10 & 600 & 10 & 1 & 69,0 & 0,010 & 4,635 & 0,43 & 8 \\
\hline 11 & 400 & 15 & 3 & 60,0 & 0,010 & 2,593 & 0,33 & 9 \\
\hline 12 & 600 & 5 & 2 & 65,0 & 0,011 & 1,702 & 0,30 & 13 \\
\hline 13 & 800 & 10 & 2 & 62,0 & 0,010 & 5,457 & 0,79 & 7 \\
\hline 14 & 400 & 10 & 3 & 59,0 & 0,011 & 2,163 & 0,30 & 13 \\
\hline 15 & 600 & 15 & 3 & 57,0 & 0,011 & 3,519 & 0,39 & 9 \\
\hline 16 & 800 & 10 & 1 & 66,0 & 0,010 & 0,586 & 0,10 & 19 \\
\hline 17 & 600 & 15 & 1 & 67,0 & 0,011 & 1,572 & 0,19 & 12 \\
\hline 18 & 600 & 5 & 1 & 69,0 & 0,774 & 5,878 & 0,41 & 11 \\
\hline 19 & 400 & 5 & 3 & 66,0 & 0,768 & 2,079 & 0,45 & 14 \\
\hline 20 & 400 & 15 & 1 & 67,0 & 0,010 & 3,178 & 0,70 & 14 \\
\hline 21 & 800 & 5 & 2 & 68,0 & 0,109 & 9,315 & 0,91 & 12 \\
\hline 22 & 600 & 15 & 2 & 62,0 & 0,092 & 9,153 & 0,91 & 10 \\
\hline 23 & 800 & 15 & 3 & 58,0 & 0,120 & 8,951 & 0,91 & 12 \\
\hline 24 & 800 & 5 & 1 & 65,0 & 0,258 & 0,275 & 0,01 & 10 \\
\hline 25 & 400 & 10 & 1 & 67,0 & 0,054 & 3,971 & 0,60 & 9 \\
\hline 26 & 600 & 10 & 2 & 65,0 & 0,101 & 11,475 & 0,89 & 7 \\
\hline 27 & 600 & 10 & 3 & 56,0 & 0,091 & 9,720 & 0,90 & 8 \\
\hline
\end{tabular}




\section{Results and Discussion}

In this study, we analyzed the impact of the following parameters: stirring speed (A), glycerin concentration (B) and sodium chloride concentration (C) in the PIT, particle size and in vitro photoprotection effectiveness of the developed nanoemulsions (Table 3).

Regarding the PIT, the results were organized in two graphs: a Pareto chart of the standardized results (Figure 1) and the residual plots (Figure 2). As discussed previously, the PIT is the temperature at which an emulsion inverts from $\mathrm{W} / \mathrm{O}$ to $\mathrm{O} / \mathrm{W}$. When this occurs, the emulsion passes through a point zero curvature and promotes a minimum surface tension, which promotes a decrease in the particle size and, consequently, the formation of nanoemulsions. Both properties improve the emulsion formation, and if the PIT is at least $20{ }^{\circ} \mathrm{C}$ above the storage temperature, there is a trend for the emulsion to be stable over time ${ }^{[20 ; 21 ; 22]}$.

The Pareto chart of the standardized results (Figure $1)$ demonstrates that the variables A $(p=0.041)$, B $(p$ $<0.001)$ and $\mathrm{C}(p<0.001)$ are significant for the PIT. $\mathrm{NaCl}$ concentration (B) has the greatest impact, followed by the glycerin concentration $(\mathrm{C})$ and the stirring speed (A). Nevertheless, the variables associations BC $(p=0.054), \mathrm{ABC}(p=0.260), \mathrm{AB}(p=0.282)$ and $\mathrm{AC}$ $(p=0.755)$ do not present statistical significance, thus they have no impact on the PIT. This result is further supported by the four residual plots (Figure 2), since the regression model is adequate and does not show the violation of the statistic assumptions ${ }^{[19]}$.

\section{Resultados e Discussão}

Neste estudo, foi analisada a influência das variáveis (velocidade de agitação, concentração de glicerina e de cloreto de sódio) na PIT, tamanho de partícula e eficácia fotoprotetora in vitro das nanoemulsões desenvolvidas (apresentadas na Tabela 2). A Tabela 3 apresenta $a$ PIT, determinação do tamanho de partícula e eficácia fotoprotetora in vitro das preparações.

Em relação à PIT, os resultados foram organizados em dois gráficos: um diagrama de Pareto dos efeitos padronizados (Figura 1) e gráficos de resíduos (Figura 2).

O diagrama de Pareto dos efeitos padronizados (Figura 1) demonstra que os fatores A $(p=0,041), \mathbf{B}(p<0,001)$ e C ( $p$ $<0,001)$ são significantes em relação à PIT enquanto os fatores $\mathbf{B C}(p=0,054), \mathbf{A B C}(p=0,260), \mathbf{A B}(p=0,282)$ e $\mathbf{A C}$ $(p=0,755)$ não apresentam significância estatística. Por conseguinte, as variáveis isoladas analisadas influenciam a PIT: $a$ concentração de $\mathrm{NaCl}$ (B) têm maior influência, seguida pela concentração de glicerina $(\mathbf{C})$ e pela velocidade de agitação (A). No entanto, a associação das variáveis $(\mathbf{B C}, \mathbf{A B C}$, $\mathbf{A B}$ e $\mathbf{A C}$ ) demonstra não influenciar a PIT. Adicionalmente, tal resultado é corroborado pelos quatro gráficos de resíduos (Figura 2), uma vez que, o modelo de regressão é adequado e não indica violação das suposições estatísticas ${ }^{[19]}$.

A temperatura em que a emulsão inverte de $\mathrm{A} / \mathrm{O}$ para O/A é chamada de PIT. Quando isto acontece, o tensoativo passa por um ponto de curvatura zero e promove a mínima tensão superficial, o que predispõe a diminuição do tamanho das partículas e consequentemente a formação de nanoemulsões ${ }^{[20]}$. Ambas propriedades beneficiam a formação de emulsão e se a PIT for ao menos $20^{\circ} \mathrm{C}$ acima da temperatura de armazenagem,

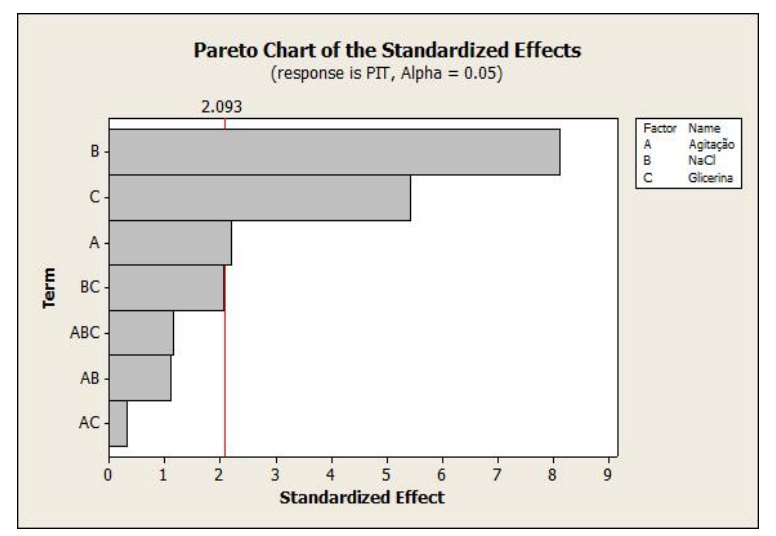

Figure 1/ Figura 1. Pareto chart of the standardized effects for PIT

Diagrama de Pareto dos efeitos padronizados para PIT 


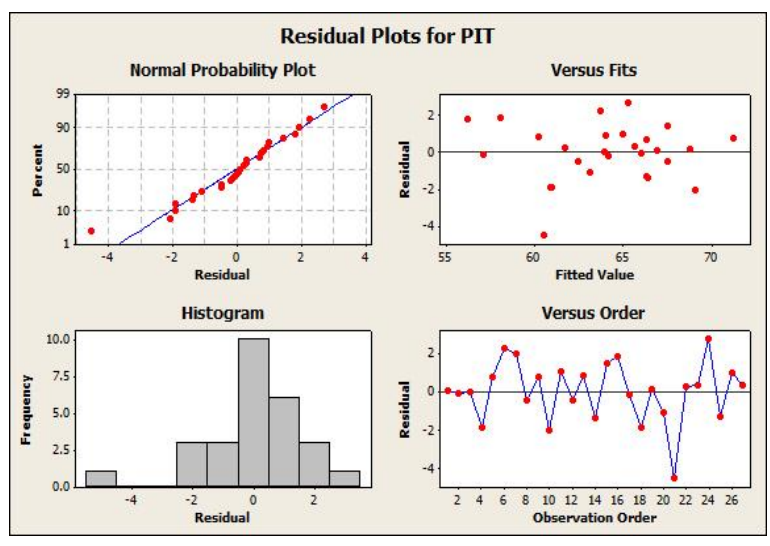

Figure 2/ Figura 2. Residual plots for PIT Gráficos de resíduos para a PIT

Regarding the particle size determination and the in vitro photoprotective effectiveness, the statistical analysis showed that the stirring speed and glycerin and sodium chloride concentrations had no impact on both parameters. The development of an effective sunscreen should consider a uniform film formation on the skin, which increases the sun protection factor. Accordingly, nanoemulsions may present a superior effectiveness when compared with conventional emulsions with regard to absorption and scattering of UV radiation. However, the particle size and the nanoparticulate system stability are parameters that must be controlled. Recent studies show that independent variables, such as a surfactant or oily compounds concentration, affect the particle size and zeta potential of nanoemulsions. Therefore, future research should be conducted in order to optimize the development of photoprotective nanoemulsions ${ }^{[23 ; 24]}$.

\section{Conclusion}

The design of experiments (DoE) was successfully used to evaluate the impact of independent variables on the development of photoprotective nanoemulsions. Statistical analysis data showed that stirring speed, as well as glycerin and sodium chloride concentrations af- há tendência de que a emulsão formada apresente estabilidade. Jiang e colaboradores (2013) indicaram que alguns eletrólitos podem diminuir a PIT de nanoemulsões. Tal propriedade é atribuída à capacidade de eletrólitos inorgânicos interagirem com tensoativos e estabilizarem a micela formada ${ }^{[20,21,22,23]}$.

Em relação à determinação do tamanho de partícula e da eficácia fotoprotetora in vitro a análise estatística indicou que as variáveis analisadas (velocidade de agitação, concentração de glicerina e de cloreto de sódio) não exerceram influência sobre os referidos parâmetros. O desenvolvimento de uma formulação fotoprotetora eficaz deve considerar a formação de uma película uniforme sobre a pele, que potencialize o fator de proteção solar. Nesse sentido, a utilização de nanoemulsões pode apresentar um perfil de eficácia superior ao das emulsões convencionais no que se refere à absorção e dispersão da radiação UV, uma vez que o tamanho reduzido das partículas favorece a formação de um filme uniforme sobre 0 relevo cutâneo. Entretanto, o tamanho de partícula, bem como a estabilidade do sistema nanoparticulado são parâmetros que devem ser controlados. Estudos recentes ${ }^{[24]}$ indicaram que variáveis independentes como a concentração de tensoativos e a concentração de compostos oleosos na formulação exercem influência em parâmetros com o tamanho de partícula e potencial zeta de nanoemulsões. Por conseguinte, observa-se que estudos futuros devem ser conduzidos com intuito de otimizar o desenvolvimento de nanoemulsões fotoprotetoras ${ }^{[24,25]}$.

\section{Conclusão}

O design de experimentos (DOE) foi utilizado com sucesso na avaliação da influência das variáveis independentes no desenvolvimento de nanoemulsões fotoprotetoras. Os resultados da análise estatística demonstrou que as variáveis (velocidade de agitação, concentração 
fect the PIT, but do not affect the particle size or the in vitro photoprotective effectiveness of the nanoemulsions. Considering the PIT, the $\mathrm{NaCl}$ concentration (B) showed the greatest impact, followed by the glycerin concentration (C) and the stirring speed (A).

\section{Conflict of Interests}

The authors declare that there are no financial and personal relationships that could be viewed as a potential conflict of interests. de glicerina e de cloreto de sódio) influenciaram a temperatura em que a emulsão inverte de $\mathrm{A} / \mathrm{O}$ para $\mathrm{O} / \mathrm{A}$ (PIT) da formulação estudada, porém não influenciaram o tamanho de partícula nem a eficácia fotoprotetora in vitro da nanoemulsão.

\section{Conflito de Interesses}

Os autores declaram não existir qualquer relação de natureza financeira ou pessoal que possa, ser entendida como, ou representar, um potencial conflito de interesses. 


\section{References / Referências}

1. Flor, J.; Davolos, M. R.; Correa, M. A. Protetores Solares. Química Nova 2007, v.30, n.1, p.153-158.

2. Andreassi, M. Sunscreens and photoprotection. Expert Rev Dermatol 2011, v.6, n.5, p.433-435.

3. ECCLESTON, G. M. Functions of mixed emulsifiers and emulsifying waxes in dermatological lotions and creams. Colloids Surfaces A: Physicochemical and Engineering Aspects 1997, v.123-124, p.169-182.

4. Pey, C. M.; Maestro, A.; Solé, I.;Gonzáles, C.; Solans, C.; Gutiérrez, J. M.; Optimization of nano emulsions prepared by low energy emulsification methods at constant temperature using a factorial design study. Colloids and Surface A: Physicochemical and Engineering Aspects 2006, v.288, p.144-150.

4. Mason, T. G.; Wilking, J. N.; Meleson, K.; Chang, C. B.; Graves, S. M. Nanoemulsions: formation, structure, and physical properties. Journal of Physics: Condensed Matter 2006, v.18, p.635-666.

5. Sonneville-Aubrun, O.; Simonnet, J.T.; L'alloret, F. Nanoemulsions: a new vehicle for skincare products. Advances in Colloid and Interface Science 2004, v.108-109, p.145-149.

6. Silva, D. G. E. ; Sarruf, F. D. ; Oliveira, L. C. D. ; Areas, E. P. G. ; Kaneko, T ; Consiglieri, V O ; Velasco, M V R; Baby, A R. Influence of particle size on appearance and in vitro efficacy of sunscreens. Brazilian Journal of Pharmaceutical Sciences (Impresso) 2013, v. 49 , p. 251-261.

7. Tadros, T.; Izquierdo, P.; Esquena, J. Formation and Stability of Nano-Emulsions. Advances in Colloid and Interface Science 2004, v.108-109, p.303-318.

8. Izquierdo, P.; Esquena, J.; Tadros, Th. F.; Dederen, C.; Garcia, M. J.; Azemar, N.; Solans, C. Formation and Stability of NanoEmulsions Prepared Using the Phase Inversion Temperature Method, Langmuir 2002, v.18, p.26-30.

9. Lin Ee, S.; Duan, X.; Liew, J.; Dzuy, N. Q. Droplet size and stability of nano-emulsions produced by the temperature phase inversion method. Chemical Engineering Journal 2008, v.140, p.626-631.
10. Sun, Y-P.; Li, X.; Cao, J.; Zhang, W.; Wang, H. P. Characterization of zero-valent iron nanoparticles. Advances in Colloid and Interface Science 2006, v.120, p.47-56.

11. Dukhin, A. S.; Goetz, P. J. Characterization of aggregation phenomena by means of acoustic and electroacoustic spectroscopy. Colloids and Surfaces A: Physicochemical and Engineering Aspects 1998, v.144, p.4958.

12. Dukhin, A. S.; Goetz, P. J. Ultrasound for characterizing colloids: particle sizing, zeta potential, rheology. New York: Elsevier Science, 2002. $372 \mathrm{p}$.

13. Bonacucina, G.; Cespi, M.; Palmieri, G.F. Evaluation of dissolution kinetics of hydrophilic polymers by use of acoustic spectroscopy. International Journal of Pharmaceutics 2009, v.377, p.153-158

14. Springsteen, A; Yurek, R; Frazier, M. Carr, K.F. In vitro measurement of sun protection factor of sunscreens by diffuse transmitance. Analitica Chimica Acta 1999, v.380, p.155164.

15. Velasco, M.V.R.; Sarruf, F.D.; Salgado-Santos, I.M.N.; Haroutiounian, C.A.; Kaneko, T.M.; Baby, A.R. Broad spectrum bioactive sunscreens. International Journal of Pharmaceutics 2008 , v.363, p.50-57.

16. Velasco, MVR, Sarruf FD, Oliveira CA, Silva APM, Consiglieri VO, Kaneko TM, Baby AR. Influence of a bioactive substance on the physicochemical and functional stability of sunscreen emulsions. Biomedical and Biopharmaceutical Research 2012; 9:119-130.

17. Barros Neto, B.; Scarminio, I.S.; Bruns, R. E. Planejamento e otimização de experimentos. $2^{\mathrm{a}}$ ed. Campinas: Editora da Unicamp, 1996. 299p.

18. Cunico, M. W. M.; Cunico, M. M.; Miguel, O. G.; Zawadzki, S. F.; Peralta-Zamora, P.; Volpato, N. Planejamento Fatorial: uma ferramenta estatística valiosa para a definição de parâmetros experimentais empregados na pesquisa científica. Visão Acadêmica 2008, v.9, n.1, p.23-32.
19. Minitab. Meet Minitab 15; for Windows. USA, 2007. 142 p. Disponível em: <http:// www.minitab.com/pt-BR/support/documentation/minitab/getting-started.asp $>$. Acesso em: 14 nov. 2012.

20. Shinoda, K.; Saito, H. The effect of temperature on the phase quilibria and the types of dispersions of the ternary system composed of water, cyclohexane and nonionic surfactant. Journal of Colloid and Interface Science 1968, v.26, p.70-74.

21. Izquierdo, P.; Esquena, J.; Tadros, TH. F.; Dederen, C.; Garcia, M. J.; Azemar, N.; Solans, C. Formation and Stability of NanoEmulsions Prepared Using the Phase Inversion Temperature Method, Langmuir 2002, v.18, p.26-30.

22. Zenitech. Emulsion Optimization by Use of Phase Inversion Temperature (PIT). Old Greenwich, CT, 2010. Disponível em: $<$ http://www.zenitech.com>. Acesso em: 09 set. 2010.

23. Jiang, J., Mei, Z., Xu, J., \& Sun, D. Effect of inorganic electrolytes on the formation and the stability of water-in-oil (W/O) emulsions. Colloids and Surfaces A: Physicochemical and Engineering Aspects 2013, $429,82-90$.

24. Musa, S. H., Basri, M., Masoumi, H. R. F., Karjiban, R. A., Malek, E. A., Basri, H., \& Shamsuddin, A. F. (2013). Formulation optimization of palm kernel oil esters nanoemulsion-loaded with chloramphenicol suitable for meningitis treatment. Colloids and Surfaces. B, Biointerfaces 2013, v. 112, p. 113-9.

25. Sood, S., Jain, K., \& Gowthamarajan, K. Optimization of curcumin nanoemulsion for intranasal delivery using design of experiment and its toxicity assessment. Colloids and Surfaces. B, Biointerfaces 2014, v. 113, p. 330-7. 